\section{Bioartificial pancreas beta test}

\section{By Kai-Jye Lou, Staff Writer}

Beta-O2 Technologies Ltd. and an international team of academic collaborators have shown that the company's bioartificial pancreas can house donor islets, supply them with oxygen and protect them from the host immune system in vivo for at least three months. ${ }^{1}$ They also demonstrated that the minimally invasive implant could normalize blood glucose levels and improve glycemic control in rat models of diabetes. The team hopes to start a trial of the implant in a single patient this year.

Islet transplantation for type 1 diabetes has seen limited use in the clinic due to the short supply of donor tissue and because islet cells have high oxygen demands that make them particularly intolerant of the hypoxic conditions encountered during isolation and transplantation.

In addition, islet transplant recipients require immunosuppressive drugs to prevent graft rejection. These drugs not only carry the risk of serious infections and malignancies but also can impair the revascularization and function of grafted islets. ${ }^{2,3}$

Finally, standard islet transplantation procedures involve using a catheter to infuse islets into the portal vein of the liver. This procedure, while minimally invasive, carries the risk of portal vein thrombosis and intraperitoneal bleeding.

For three years, Beta-O2 and their collaborators have been developing a bioartificial pancreas to address the shortcomings of islet transplantation and have now published the first data showing how the system works.

The subcutaneous implant consists of two chambers separated by an oxygen-permeable membrane, with one module to house and protect an alginate slab that contains the islets and a gas module to supply the islets with oxygen (see Figure 1, "Beta-O2's bioartificial pancreas system").

In a rat model of streptozotocin-induced diabetes, implantation of a device containing rat islets normalized blood glucose levels until the implant was removed at day 90. The implant site had prominent vascularization and showed no signs of compatibility issues such as inflammation or fibrosis.

The researchers then examined the implant itself to confirm the absence of inflammatory reactions and showed that the islets housed within the alginate slab remained intact.

To tackle the problem of a limited supply of donor islets, the group looked to a 2010 paper that showed the synthetic growth hormonereleasing hormone (GHRH) agonist JI-36 could promote the survival and proliferation of pancreatic islets and also improve their ability to restore blood glucose levels and glycemic control in diabetic mice. ${ }^{4}$

Indeed, when the researchers pretreated islets with JI-36, the total number of islets needed in the implant to achieve normal blood glucose levels in diabetic rats was reduced by about $50 \%$. Rats receiving the pretreated islets also showed better glycemic control than those that received untreated islets.

Results were reported in the Proceedings of the National Academy of Sciences.

Stefan Bornstein said patients with type 1 diabetes usually need about 500,000 islets to achieve insulin independence but pointed out the current bioartificial pancreas system only houses around half that amount.

"This is why we sought to use GHRH agonists to improve the function of the transplanted islets, though 250,000 islets should be sufficient to achieve insulin independence in lean patients," said Bornstein, a coauthor of the paper who is chair of medicine and director of the University Hospital of Carl Gustav Carus and the Dresden University of Technology. "GHRH agonists could help reduce the number of islets needed, and we didn't want to make our system too big as it would complicate the implantation process."

Bornstein added that the implant's subcutaneous delivery route should pose fewer safety risks than standard islet transplantation and simplifies the process of implanting and extracting the device from the patient.

\section{$\mathrm{O}_{2}$ delivery}

"The nice thing about their system is that they incorporate an oxygen delivery system to support the transplanted islets before the graft is vascularized," said Emmanuel Opara, co-director of the islet transplantation program and a professor at the Institute for Regenerative Medicine and the Center for Diabetes Research at the Wake Forest School of Medicine. "It takes about 7-10 days for new vasculature to form around a graft, and during this time a lot of the implanted islet cells could otherwise die."

"For me, the most interesting aspect of the current study is the use of the GHRH agonist," said ViaCyte Inc. CSO Kevin D’Amour. "An adjunctive therapy like this has the potential to improve islet delivery."

ViaCyte's lead program is Pro-Islet, a preclinical, subcutaneously delivered cell therapy that provides replacement islet cells for patients with diabetes.

D'Amour said ViaCyte's strategy for addressing the oxygen supply problem is to transplant encapsulated pancreatic progenitors, which have much lower oxygen requirements than mature islet cells.

"The cells we deliver differentiate and mature into highly functional insulin-producing cells in the weeks and months after delivery, when the graft has become fully vascularized," he told SciBX. 


\section{ANALYSIS}
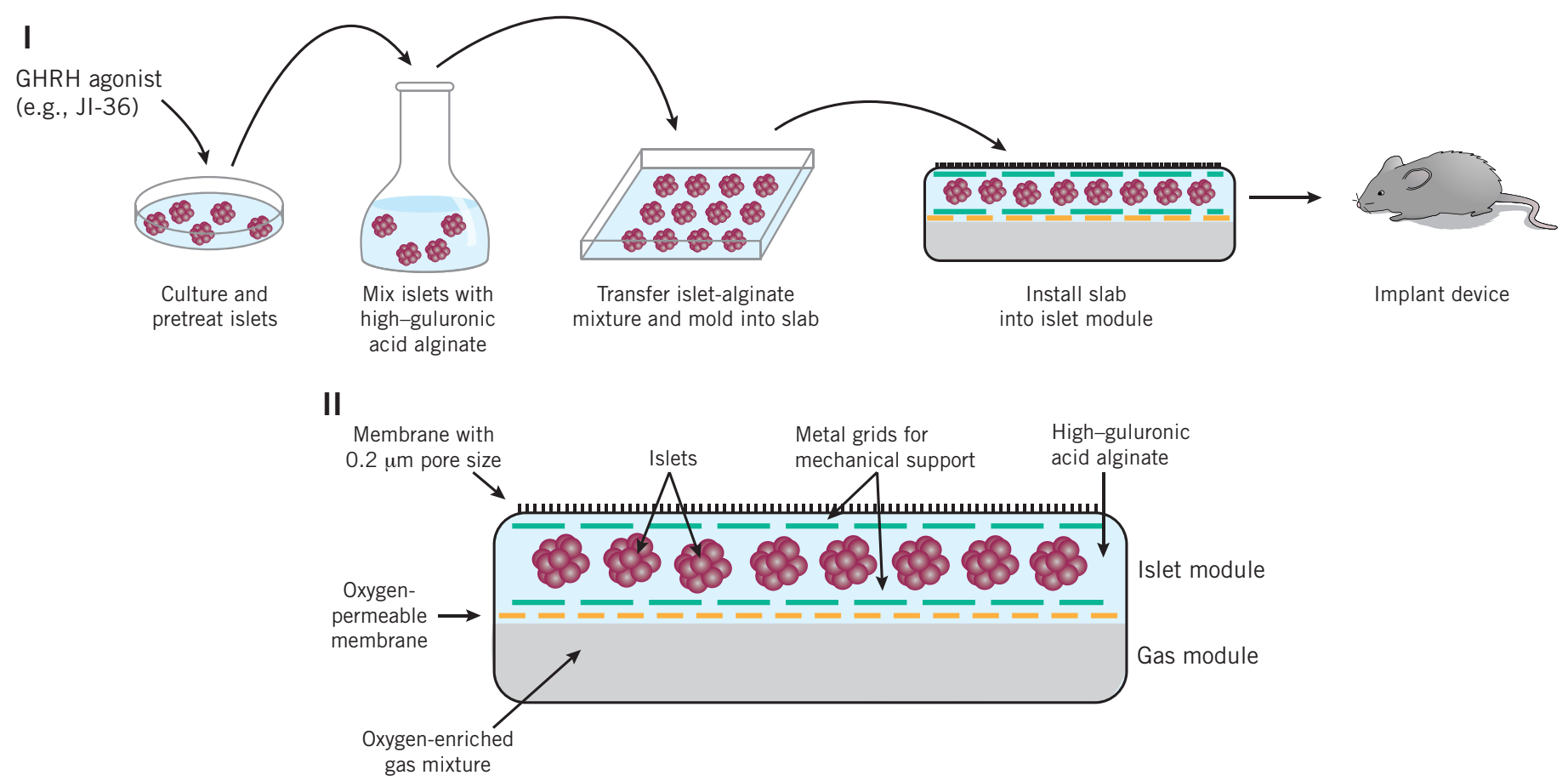

Figure 1. Beta-02's bioartificial pancreas system. As reported by Ludwig et al., the bioartificial pancreas system from Beta-02 Technologies Ltd. and collaborators restores normal blood glucose levels and improves glycemic control in rat models of diabetes.

Isolated islets are cultured in a medium containing the growth hormone-releasing hormone (GHRH) agonist $\mathrm{JI}-36$ (I). Islets are collected, mixed with a solution of high-guluronic acid alginate and transferred into a mold to create an islet-containing alginate slab. Next, the slab is installed into the islet module of the bioartificial pancreas and implanted into the host.

The porous outer membrane of the islet module and the alginate create an immune barrier to protect the islets from the host immune system while still allowing secreted factors such as insulin to pass through (II). The gas module supplies the islets with oxygen through the oxygen-permeable membrane. The metal grid in the islet module provides mechanical support for the implant. (Figure based on Figure 1 in ref. 1.)

Merck KGaA already markets the GHRH agonist Egrifta tesamorelin to help reduce excess abdominal fat in HIV-infected patients with lipodystrophy. The pharma acquired U.S. rights to the drug from Theratechnologies Inc.

Akela Pharma Inc. has a GHRH analog in Phase II testing to treat malnutrition in patients with stage IV chronic renal failure.

\section{Duration of effect}

Although the data reported in the paper demonstrate the implant's effect in rats for 90 days, the researchers still need to test the implant's longterm durability and its ability to restore glycemic control in additional animal models.

"The reported findings are mechanistically interesting, and this group has made some advances in supplying the transplanted islets with oxygen," said Matthias von Herrath, director of the Center for Type 1 Diabetes Research at the La Jolla Institute for Allergy \& Immunology and VP at the Novo Nordisk A/S type 1 diabetes R\&D center. "However, they will need to run additional studies to evaluate the implant's duration of effect. They will need to show that the implant remains functional for a year or longer for such a system to be considered viable for use in humans."
Avi Rotem, CTO of Beta-O2, said the team has run implantation experiments in rats out to 250 days.

"Upon retrieval of the device the blood glucose was returned to the disease state, like all other studies, and the islet functionality remained similar to islets at time zero," he told SciBX. "These results suggest longterm viability and functionality of the islets within Beta-O2's device is due to its built-in oxygen supply and immune protection."

ViaCyte's D'Amour wanted to see studies showing that the system can deliver human islets-the researchers used rat islets. "The researchers also suggest that their bioartificial pancreas system provides xenoprotection, so I think it would be a good idea for them to test whether their system could deliver porcine islets into immunocompetent rodents," he added.

Opara said the system should be tested in an autoimmune model of type 1 diabetes in which the animal displays other features of the disease such as autoantibodies that target islet $\beta$ cells. "One of the things I was curious about is whether the membrane surrounding the islets, which has pore size of 200 nanometers, will be able to block some of the molecules that could attack the implanted islets, such as antibodies," he told SciBX.

Bornstein acknowledged that the bioartificial pancreas may need 


\section{ANALYSIS}

further optimization, such as increasing the amount of oxygen the system delivers to the islets.

Rotem said the group is now testing the bioartificial pancreas in a diabetic pig model.

"We will be ready to test our system in human trials very soon, and we will most likely

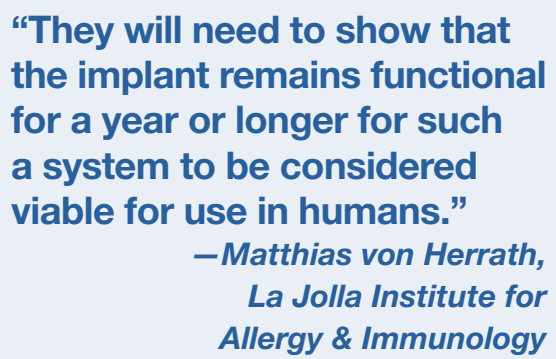

"They will need to show that the implant remains functional for a year or longer for such a system to be considered viable for use in humans." -Matthias von Herrath, La Jolla Institute for Allergy \& Immunology start by using our system to deliver human islets," added Bornstein. "However, I do see the potential to use this system to deliver porcine islets in future trials. Currently, we hope to start a single-patient trial later this year and then, depending on the results of these trials, start a larger clinical trial in the next two to three years."

Andrew Schally, corresponding author on the PNAS paper, said his group has developed more potent GHRH analogs than JI-36 and plans to evaluate the effects of these newer analogs on islet function in future studies.

Schally is a medical research scientist of the U.S. Department of Veterans Affairs and head of the Endocrine, Polypeptide and Cancer Institute at the Miami VA Healthcare System. He also is a professor in the department of medicine at the University of Miami Miller School of Medicine.

The University of Miami and the U.S. Department of Veterans
Affairs have filed a patent covering GHRH agonists for promoting the survival and growth of pancreatic islets. The patent is available for licensing. Rotem said Beta-O2 has filed multiple patents covering its bioartificial pancreas and said the company is interested in finding a partner to help develop the system.

Lou, K.-J. SciBX 5(12); doi:10.1038/scibx.2012.298

Published online March 22, 2012

\section{REFERENCES}

1. Ludwig, B. et al. Proc. Natl. Acad. Sci. USA; published online March 5, 2012; doi:10.1073/pnas.1201868109

Contact: Andrew V. Schally, University of Miami Miller School of Medicine, Miami, Fla.

e-mail: andrew.schally@va.gov

2. Hyder, A. et al. Toxicol. In Vitro 19, 541-546 (2005)

3. Lohmann, T. et al. Exp. Clin. Endocrinol. Diabetes 108, 347-352 (2000)

4. Ludwig, B. et al. Proc. Natl. Acad. Sci. USA 107, 12623-12628 (2010)

COMPANIES AND INSTITUTIONS MENTIONED

Akela Pharma Inc. (TSX:AKL), Austin, Texas

Beta-02 Technologies Ltd., Petah Tikva, Israel Dresden University of Technology, Dresden, Germany La Jolla Institute for Allergy \& Immunology, La Jolla, Calif. Merck KGaA (Xetra:MRK), Darmstadt, Germany Miami VA Healthcare System, Miami, Fla. Novo Nordisk A/S (CSE:NVO; NYSE:NVO), Bagsvaerd, Denmark Theratechnologies Inc. (TSX:TH; NASDAQ:THER), Montreal, Quebec, Canada

University Hospital of Carl Gustav Carus, Dresden, Germany University of Miami, Miami, Fla.

University of Miami Miller School of Medicine, Miami, Fla. U.S. Department of Veterans Affairs, Washington, D.C. ViaCyte Inc., San Diego, Calif.

Wake Forest School of Medicine, Winston-Salem, N.C. 